\title{
One-pot Green Synthesis for Pyrimido[4,5-d]pyrimidine Derivatives
}

\author{
Mazaahir Kidwai, Kavita Singhal, and Shuchi Kukreja \\ Green Chemistry Research Laboratory, Department of Chemistry, University of Delhi, Delhi-10007, \\ India \\ Reprint requests to Prof. M. Kidwai. Fax: (+91 11) 27666235. E-mail: kidwai.chemistry@ gmail.com
}

Z. Naturforsch. 2007, 62b, 732-736; received November 11, 2006

\begin{abstract}
Water has been used efficiently as a solvent for the reaction of barbituric acid, aldehyde and urea or thiourea to yield pyrimido[4,5- $d]$ pyrimidines. This environmentally benign procedure leads to high yields of products of greater purity in a single step using water.
\end{abstract}

Key words: Barbituric Acid, Pyrimido[4,5- $d$ ]pyrimidines, Water, Multicomponent Reaction

\section{Introduction}

For the synthetic chemists, water often appears as a natural enemy to be kept away from the reaction mixture until workup. However, recently water [1] has been reported as a medium in various transformation reactions with a considerable rate increase [2]. This has been explained on the basis of its permanent dipole moment; hence at elevated temperature, water facilitates the isolation of products due to the decreased solubility of organic materials. Further reasons to make water unique as compared to organic solvents are its cheapness and non-inflammability, putting water in the domain of green solvents.

Heterocyclic chemistry is currently experiencing a renaissance because of the interest in heterocyclic scaffolds as templates for combinatorial chemistry [3]. As heteroaromatic compounds are present in many natural products [4], and are constituents of numerous therapeutic agents [5], they represent ideal drug-like structures for the elaboration of and increase in molecular diversity.

Multicomponent reactions (MCRs) have been proved as simple and convenient ways to produce a plethora of physiologically active pyrimidinones [6]. Three-component reactions such as the Biginelli reaction with cyclic 1,3-dicarbonyl compounds, aromatic aldehydes and urea/thiourea are well known microwave-induced reactions (MW) [7]. Pyrimidines and fused pyrimidine derivatives represent a class of annulated uracils with biological significance because of their close association with purines and pteridine systems [8]. Numerous reports have been patented [9] and have delineated the antiallergic [10], antiviral [11], antibacterial [12], antioxidant [13] and hepatoprotective properties [14] of fused pyrimidines. The promising methods for the synthesis of pyrimido[4,5- $d$ ]pyrimidines involve multistep syntheses starting from 1,3-disubstituted cyanouracils [15], polymer bound 2-(alkylsulfanyl)-4-aminopyrimidine5-carbonitriles [16], aza-Wittig-type reactions using iminophosphoranes of 5-aminouracils [17], and reacting 6-[(dimethylamino)methylene]aminouracils with various heterocumulenes [18]. Synthetic alternatives are many and varied and have resorted to harsh conditions, $e . g$. the use of PTSA ( $p$-toluenesulfonic acid) as catalyst, using $\mathrm{POCl}_{3}$ with DMF as a solvent [19]. Additionally, reagents for these procedures are not readily or commercially available which is a key deficiency in developing conditions for library synthesis.

Moreover, water-mediated single-flask procedures for the synthesis of pyrimido[4,5- $d$ ] pyrimidines avoiding harmful catalysts have not been reported yet.

As part of our ongoing program of developing environmentally benign synthetic methods [20] for pharmacologically important heterocyclic skeletons, we explored a green multicomponent reaction (MCR) protocol using water as solvent. Herein, we report a simple, rapid and one-pot water-mediated procedure for the synthesis of pyrimido[4,5- $d]$ pyrimidines in high yield and purity.

\section{Results and Discussion}

Choosing an appropriate solvent is of crucial importance for the successful microwave-assisted synthesis. To search for the optimal solvent, the reaction of barbituric acid $(\mathrm{BA}, 1)(0.01 \mathrm{mmol})$, aromatic aldehydes 
Table 1. Solvent optimization for the synthesis of compound 4f under microwave irradiation conditions.

\begin{tabular}{lllll}
\hline Entry & Solvent & Power $(\mathrm{W})$ & Time $(\mathrm{min})$ & Yield (\%) \\
\hline 1. & THF & 400 & - & - \\
2. & Ethanol & 400 & 7.0 & 65 \\
3. & DMF & 400 & 6.5 & 71 \\
4. & Acetic acid & 400 & 6.0 & 67 \\
5. & Glycol & 400 & 4.5 & 87 \\
6. & Water & 400 & 4.0 & 88 \\
7. & Water & 560 & 2.3 & 92 \\
\hline
\end{tabular}

$$
\mathrm{N}_{\mathrm{NH}}^{\mathrm{Na}-\mathrm{g}}
$$

$$
\begin{aligned}
& \mathbf{R} \\
& \mathbf{2 a}=\text { Phenyl } \\
& \mathbf{2 b}=\text { 2-Hydroxyphenyl } \\
& \mathbf{2 c}=\text { 4-Methoxyphenyl } \\
& \mathbf{2 d}=\text { 4-Chlorophenyl } \\
& \mathbf{2 e}=\text { 1,3-Benzodioxol-5-yl } \\
& \mathbf{2 f}=\text { 2-Thienyl } \\
& \mathbf{2 g}=\text { 2-Chloroquinolinyl }
\end{aligned}
$$

Scheme 1. One-pot synthesis of pyrimido[4,5-d]pyrimidinetriones.

$2 \mathbf{a}-\mathbf{g}(0.01 \mathrm{mmol})$ and urea (3a) $(0.01 \mathrm{mmol})$ was examined using water, glycol, DMF, THF or ethanol as solvents under MW irradiation conditions. Some of the reactions were also performed at different powers of microwaves. As shown in Table 1, the reaction proceeded efficiently when water was used as solvent resulting in higher yields in comparison to the other solvents used. Inspired by this result, water was then used as solvent for all further MW-assisted reactions.

To optimize the reaction temperature, the synthesis of $4 \mathbf{f}$ was performed in water at temperatures ranging from 70 to $130{ }^{\circ} \mathrm{C}$, at different powers of microwaves, i.e. 400, 480, 560, 640, $800 \mathrm{~W}$. At low powers $(400,480 \mathrm{~W})$, the time taken for the temperature to reach $100{ }^{\circ} \mathrm{C}$ was too long. Microwave irradiation at $560 \mathrm{~W}$ in $2-3 \mathrm{~mL}$ of water furnished the highest yield of 5-thienyl-1,3,5,6,8-pentahydropyrimido[4,5- $d$ ]pyrimidine-2,4,7-trione $4 \mathbf{f}$ (Scheme 1), without evaporation of the solvent. Thus, a microwave power of $560 \mathrm{~W}$ was chosen as the optimal one. The ease of separation of pyrimido[4,5- $d]$ pyrimidine derivatives from the reaction media is the foremost advantage of this process in aqueous media. Similarly, thiourea (3b) $(0.01 \mathrm{mmol})$ in place of urea was taken (Scheme 2) to get 5-aryl-1,3,5,6,8-pentahydro7-thioxo-pyrimido[4,5- $d$ ] pyrimidine-2,4-diones $\mathbf{6 a - f . ~}$ Thus thiourea proved here as one of the ingredi-
Table 2. Comparison of reaction times and yields of compounds $4 \mathbf{a}-\mathbf{g}$ and $6 \mathbf{a}-\mathbf{g}$.

\begin{tabular}{llllll}
\hline Comp. & R & \multicolumn{2}{c}{ Method A } & \multicolumn{2}{c}{ Method B } \\
& & Time & Yield & Time & Yield \\
& & $(\mathrm{h})$ & $(\%)$ & $(\mathrm{min})$ & $(\%)$ \\
\hline $\mathbf{4 a}$ & Phenyl & 2.0 & 80 & 2.3 & 87 \\
$\mathbf{4 b}$ & 2-Hydroxyphenyl & 3.5 & 70 & 3.3 & 82 \\
$\mathbf{4 c}$ & p-Methoxyphenyl & 4.0 & 76 & 2.0 & 85 \\
$\mathbf{4 d}$ & 4-Cl-phenyl & 4.0 & 72 & 3.0 & 86 \\
$\mathbf{4 e}$ & Piperonyl & 3.5 & 72 & 2.0 & 85 \\
$\mathbf{4 f}$ & 2-Thienyl & 3.0 & 80 & 2.3 & 92 \\
$\mathbf{4 g}$ & 2-Cl-3-Quinolinyl & 4.5 & 76 & 3.3 & 87 \\
$\mathbf{6 a}$ & Phenyl & 1.5 & 80 & 2.3 & 90 \\
$\mathbf{6 b}$ & 2-Hydroxyphenyl & 4.0 & 74 & 3.0 & 80 \\
6c & p-Methoxyphenyl & 2.0 & 75 & 2.3 & 88 \\
6d & 4-Cl-phenyl & 2.5 & 80 & 3.0 & 92 \\
6e & Piperonyl & 2.5 & 70 & 1.3 & 88 \\
6f & 2-Thienyl & 3.5 & 76 & 2.3 & 90 \\
\hline
\end{tabular}<smiles>[R]C1NC(=S)Nc2[nH]c(=O)[nH]c(=O)c2C1[2H]</smiles>

$\mathbf{R}$
$=$ Phenyl

$2 \mathbf{b}=2$-Hydroxyphenyl

$2 \mathrm{c}=4$-Methoxyphenyl

$\mathbf{2 c}=4$-Methoxypheny
$\mathbf{2 d}=4$-Chlorophenyl

$\mathbf{2 d}=4$-Chlorophenyl
$\mathbf{2 e}=1,3$-Benzodioxol-5-yl

$\mathbf{2 e}=1,3$-Benzo
$\mathbf{2 f}=2$-Thienyl

Scheme 2. Synthesis of 7-thioxo-pyrimido[4,5- $d]$ pyrimidine-diones.

ents with similar success to provide the corresponding pyrimido $[4,5-d]$ pyrimidine thiones which are also of interest with respect to their biological activities. Likewise, 2-thiobarbituric acid (TBA) can also be employed as an alternative under these aqueous conditions.

Moreover, for comparison, classical heating conditions were also applied for the synthesis of all products as is summarized in Table 2. It was found that the reaction proceeds efficiently under MW irradiation with a reduction in time from hours to minutes in comparison to the conventional one with appreciable yield enhancement. In addition, looking at the success of this approach, as no extra catalyst was required for the proposed protocol, it was thought worthwhile to attempt the reactions without any solvent or catalyst, but no proper results were obtained; only some charring was observed on prolonged heating. This emphasizes the importance of water which acts as a solvent and provides a media for the transfer of energy. Thus an example has been established for the condensation reaction in water. 
The structures of products $4 \mathbf{a}-\mathbf{g}$ and $\mathbf{6 a}-\mathbf{f}$ as pyrimido[4,5- $d]$ pyrimidinones were assigned on the basis of the spectral data and the elemental analyses. In the ${ }^{1} \mathrm{H}$ NMR spectra, the disappearance of the diagnostic signal due to the methylene protons of barbituric acid at $\delta=3.8 \mathrm{ppm}$ indicates the condensation of the active methylene group with the aromatic aldehydes used. Following the mechanism, this intermediate then condenses with urea/thiourea furnishing the required products $4 \mathbf{a}-\mathbf{g}$ and $\mathbf{6 a}-\mathbf{f}$, which is confirmed by the appearance of a singlet at $\delta=4.9 \mathrm{ppm}$ assigned to $5-\mathrm{H}$.

In brief, a practically convenient and eco-friendly synthesis of pyrimido[4,5- $d$ ]pyrimidines has been developed in an aqueous medium without using any catalyst, i.e. completely circumventing the use of hazardous organic solvents and corrosive acids or bases. Water-insoluble solid products obtained in short time are found to be essentially pure and in very high yield. This simple single-step reaction has the ability to withstand the variations in the 1,3-diketone and carbonyl part also. This direct strategy could find broader interest for the synthesis of compound libraries having in common a heterocyclic pyrimido[4,5- $d$ ] pyrimidine moiety.

\section{Experimental Section}

Melting points were taken on a Thomas Hoover melting point apparatus and are uncorrected. IR spectra (in nujol) were recorded on a model Perkin-Elmer FTIR-1710 spectrophotometer, ${ }^{1} \mathrm{H}$ NMR spectra on a Bruker Avance Spectrospin 300 instrument $(300 \mathrm{MHz})$ using TMS as internal standard. Elemental analyses were performed on a Heraeus CHN Rapid Analyzer. Mass spectra were recorded on a KC455 Waters TOF spectrometer. The purity of the compounds was checked on silica gel-coated aluminum plates (Merck). A Kenstar microwave oven, Model No. OM9925E $(2450 \mathrm{MHz}, 800 \mathrm{~W})$ was used for microwave-assisted reactions. The temperature of the reaction mixture was measured with an AZ, mini gun-type, non-contact IR thermometer, model no. 8868 .

General procedure for the synthesis of 5-aryl-1,3,5,6,8pentahydropyrimido[4,5-d] pyrimidine derivatives

Method A (conventional synthesis)

To the mixture of barbituric acid $(\mathbf{1}, 0.01 \mathrm{mmol})$, an aromatic aldehyde $(\mathbf{2 a}-\mathbf{g}, 0.01 \mathrm{mmol})$ and urea/thiourea $(\mathbf{3 a} / \mathbf{3 b}$, $0.01 \mathrm{mmol}), 10 \mathrm{~mL}$ of water were added. The reaction mixture was kept at reflux for an appropriate time with constant stirring. The solid obtained was isolated by filtration, washed with water and dried.

\section{Method B (microwave-assisted synthesis)}

An Erlenmeyer flask was charged with equimolar amounts of barbituric acid (1, $0.01 \mathrm{mmol})$, an aromatic aldehyde $(\mathbf{2} \mathbf{a}-\mathbf{g}, 0.01 \mathrm{mmol})$, urea/thiourea (3a/3b, $0.01 \mathrm{mmol})$, and $2-3 \mathrm{~mL}$ of water. The reaction mixture was subjected to microwave irradiation for a specific time (Table 1) at low power $(560 \mathrm{~W})$. The progress of the reaction was monitored at intervals of $30 \mathrm{sec}$. Upon completion, the reaction mixture was cooled and the product was isolated by filtration, washed with cold water and dried.

5-Phenyl-1,3,5,6,8-pentahydro-pyrimido[4,5-d]pyrimidine2,4,7-trione ( $4 a)$

White solid; m.p. $244-246{ }^{\circ} \mathrm{C}$; anal. calcd. for $\mathrm{C}_{12} \mathrm{H}_{10} \mathrm{~N}_{4} \mathrm{O}_{3}$ : C 55.81, H 3.87, N 21.70; found: C 55.86, H 3.79, N 21.75. - IR (nujol): $v=3480,3213,3083$, 1749, 1678, 1648, 1583, $1565 \mathrm{~cm}^{-1} .-{ }^{1} \mathrm{H}$ NMR $(300 \mathrm{MHz}$, $\left.\mathrm{CDCl}_{3}+\left[\mathrm{D}_{6}\right] \mathrm{DMSO}\right): \delta=11.27(\mathrm{~s}, 1 \mathrm{H}, \mathrm{NH}), 10.99(\mathrm{~s}, 2 \mathrm{H}$, $\mathrm{NH}), 8.26(\mathrm{~s}, 1 \mathrm{H}, \mathrm{NH}), 7.03-7.27(\mathrm{~m}, 5 \mathrm{H}$, arom. $\mathrm{H}), 4.88(\mathrm{~s}$, $1 \mathrm{H}, 5-\mathrm{H}) .-\mathrm{MS}: m / z=259[\mathrm{M}+\mathrm{H}]^{+}$.

\section{5-(2-Hydroxyphenyl)-1,3,5,6,8-pentahydro-pyrimido- [4,5-d]pyrimidine-2,4,7-trione (4b)}

White solid; m.p. $220-222{ }^{\circ} \mathrm{C}$; anal. calcd. for $\mathrm{C}_{12} \mathrm{H}_{10} \mathrm{~N}_{4} \mathrm{O}_{4}$ : C 52.55, H 3.64, N 20.43; found: C 52.49, H 3.61, N 20.35. - IR (nujol): $v=3486,3279,3220$, 3120, 1748, 1695, 1651, $1581 \mathrm{~cm}^{-1} .-{ }^{1} \mathrm{H}$ NMR $(300 \mathrm{MHz}$, $\left.\mathrm{CDCl}_{3}+\left[\mathrm{D}_{6}\right] \mathrm{DMSO}\right): \delta=1.26(\mathrm{~s}, 1 \mathrm{H}, \mathrm{NH}), 10.99(\mathrm{~s}, 2 \mathrm{H}$, $\mathrm{NH}), 8.29$ (s, 1H, NH), $7.21-7.34$ (m, 4H, arom. H), 4.91 (s, 1H, 5-H). $-\mathrm{MS}: m / z=274[\mathrm{M}+\mathrm{H}]^{+}$.

\section{5-(4-Methoxyphenyl)-1,3,5,6,8-pentahydro-pyrimido- [4,5-d]pyrimidine-2,4,7-trione $(4 \mathrm{c})$}

White solid; m.p. $284-286{ }^{\circ} \mathrm{C}$; anal. calcd. for $\mathrm{C}_{13} \mathrm{H}_{12} \mathrm{~N}_{4} \mathrm{O}_{4}$ : C 54.16, H 4.16, N 19.44; found: C 54.09, H 4.22, N 19.40. - IR (nujol): $v=3468,3283,3069$, 1756, 1693, 1655, $1548 \mathrm{~cm}^{-1} .{ }^{1} \mathrm{H}$ NMR $\left(300 \mathrm{MHz}, \mathrm{CDCl}_{3}+\right.$ [D $\mathrm{D}_{6}$ DMSO): $\delta=11.29$ (s, 1H, NH), 10.97 (s, 2H, NH), 8.31 (s, 1H, NH), 7.20 (d, $J=7.0 \mathrm{~Hz}, 2 \mathrm{H}$, arom. H), 7.14 (d, $J=7.0 \mathrm{~Hz}, 2 \mathrm{H}$, arom. H), $4.90(\mathrm{~s}, 1 \mathrm{H}, 5-\mathrm{H}), 3.81$ (s, $3 \mathrm{H}$, $\left.\mathrm{OCH}_{3}\right) .-\mathrm{MS}: m / z=289[\mathrm{M}+\mathrm{H}]^{+}$.

\section{5-(4-Chlorophenyl)-1,3,5,6,8-pentahydro-pyrimido[4,5-d]-} pyrimidine-2,4,7-trione $(\mathbf{4 d})$

White solid; m.p. 296-298 ${ }^{\circ} \mathrm{C}$; anal. calcd. for $\mathrm{C}_{12} \mathrm{H}_{9} \mathrm{~N}_{4} \mathrm{O}_{3} \mathrm{Cl}$ : C 49.23, H 3.07, N 19.14; found: C 49.31, H 3.18, N 19.06. - IR (nujol): $v=3484,3223,3090$, 
1760, 1705, 1673, 1644, $1575 \mathrm{~cm}^{-1} .-{ }^{1} \mathrm{H}$ NMR (300 MHz, $\left.\mathrm{CDCl}_{3}+\left[\mathrm{D}_{6}\right] \mathrm{DMSO}\right): \delta=11.27(\mathrm{~s}, 1 \mathrm{H}, \mathrm{NH}), 10.98(\mathrm{~s}, 2 \mathrm{H}$, $\mathrm{NH}), 8.26(\mathrm{~s}, 1 \mathrm{H}, \mathrm{NH}), 7.61(\mathrm{~d}, J=8.0 \mathrm{~Hz}, 2 \mathrm{H}$, arom. H), $7.26(\mathrm{~d}, J=8.0 \mathrm{~Hz}, 2 \mathrm{H}$, arom. H), $4.90(\mathrm{~s}, 1 \mathrm{H}, 5-\mathrm{H}) .-\mathrm{MS}$ : $m / z=292.03[\mathrm{M}]^{+}$.

5-(1,3-Benzodioxol-5-yl)-1,3,5,6,8-pentahydro-pyrimido [4,5-d]pyrimidine-2,4,7-trione (4e)

Yellow solid; m.p. $>300{ }^{\circ} \mathrm{C}$; anal. calcd. for $\mathrm{C}_{13} \mathrm{H}_{10} \mathrm{~N}_{4} \mathrm{O}_{5}$ : C 51.65, H 3.31, N 18.54 found: C 51.54, H 3.38, N 18.61. - IR (nujol): $v=3480,3273,3090,1760$, 1696, 1635, $1575 \mathrm{~cm}^{-1} .-{ }^{1} \mathrm{H}$ NMR $\left(300 \mathrm{MHz}, \mathrm{CDCl}_{3}+\right.$ [D $\mathrm{D}_{6}$ DMSO): $\delta=11.27(\mathrm{~s}, 1 \mathrm{H}, \mathrm{NH}), 10.98(\mathrm{~s}, 2 \mathrm{H}, \mathrm{NH})$, $8.30(\mathrm{~s}, 1 \mathrm{H}, \mathrm{NH}), 7.11(\mathrm{~s}, 1 \mathrm{H}$, arom. H), $6.87(\mathrm{~d}, J=8.0 \mathrm{~Hz}$, $1 \mathrm{H}$, arom. $\mathrm{H}), 6.71(\mathrm{~d}, J=8.0 \mathrm{~Hz}, 1 \mathrm{H}$, arom. $\mathrm{H}), 5.93(\mathrm{~s}, 2 \mathrm{H}$, $\left.\mathrm{OCH}_{2} \mathrm{O}\right), 4.90(\mathrm{~s}, 1 \mathrm{H}, 5-\mathrm{H}) .-\mathrm{MS}: m / z=303[\mathrm{M}+\mathrm{H}]^{+}$.

\section{5-(2-Thienyl)-1,3,5,6,8-pentahydro-pyrimido[4,5-d]- pyrimidine-2,4,7-trione $(\mathbf{4 f})$}

Yellow solid; m.p. $>280{ }^{\circ} \mathrm{C}$; anal. calcd. for $\mathrm{C}_{10} \mathrm{H}_{8} \mathrm{~N}_{4} \mathrm{O}_{3} \mathrm{~S}$ : C 45.45, H 3.03, N 21.21; found: C 45.50, H 3.01, N 21.33. - IR (nujol): $v=3472,3146,3062,1756$, 1683, 1655, $1558 \mathrm{~cm}^{-1} .{ }^{1} \mathrm{H}$ NMR $\left(300 \mathrm{MHz}, \mathrm{CDCl}_{3}+\right.$ [D $\mathrm{D}_{6}$ DMSO): $\delta=11.28(\mathrm{~s}, 1 \mathrm{H}, \mathrm{NH}), 10.96(\mathrm{~s}, 2 \mathrm{H}, \mathrm{NH})$, $8.40(\mathrm{~d}, J=4.6 \mathrm{~Hz}, 1 \mathrm{H}$, thienyl-H), 8.25 (s, $1 \mathrm{H}, \mathrm{NH}), 8.14$ (d, $J=3.0 \mathrm{~Hz}, 1 \mathrm{H}$, thienyl-H), 7.48 (t, $1 \mathrm{H}$, thienyl-H), 4.90 (s, 1H, 5-H). - MS: $m / z=265[\mathrm{M}+\mathrm{H}]^{+}$.

\section{5-(2-Chloroquinolinyl)-1,3,5,6,8-pentahydro-pyrimido [4,5-d]pyrimidine-2,4,7-trione $\mathbf{( 4 g})$}

Yellow solid; m.p. $>280{ }^{\circ} \mathrm{C}$; anal. calcd. for $\mathrm{C}_{10} \mathrm{H}_{8} \mathrm{~N}_{4} \mathrm{O}_{3} \mathrm{~S}$ : C 56.56, H 3.70, N 23.56; found: C 56.63, H 3.81, N 23.46. - IR (nujol): $v=3480,3283,3094,1754$, 1698, 1640, $1563 \mathrm{~cm}^{-1} .-{ }^{1} \mathrm{H}$ NMR $\left(300 \mathrm{MHz}, \mathrm{CDCl}_{3}+\right.$ [D $\mathrm{D}_{6}$ DMSO): $\delta=11.29(\mathrm{~s}, 1 \mathrm{H}, \mathrm{NH}), 10.98(\mathrm{~s}, 2 \mathrm{H}, \mathrm{NH})$, $8.26(\mathrm{~s}, 1 \mathrm{H}, \mathrm{NH}), 7.68-7.74(\mathrm{~m}, 3 \mathrm{H}$, arom. $\mathrm{H}), 4.90(\mathrm{~s}, 1 \mathrm{H}$, 5-H). - MS: $m / z=298[\mathrm{M}+\mathrm{H}]^{+}$.

\section{5-Phenyl-1,3,5,6,8-pentahydro-7-thioxo-pyrimido[4,5-d]- pyrimidine-2,4-dione ( $6 a$ )}

White solid; m.p. 290-292 ${ }^{\circ} \mathrm{C}$; anal. calcd. for $\mathrm{C}_{12} \mathrm{H}_{10} \mathrm{~N}_{4} \mathrm{O}_{2} \mathrm{~S}$ : C 48.0, $\mathrm{H} 4.0, \mathrm{~N} 22.40$; found: C 48.12, $\mathrm{H}$ 3.96, N 22.34. - IR (nujol): $v=3452,3195,1705$, 1680, 1657, $1564 \mathrm{~cm}^{-1} .{ }^{1} \mathrm{H}$ NMR $\left(300 \mathrm{MHz}, \mathrm{CDCl}_{3}+\right.$ [D $\mathrm{D}_{6}$ DMSO): $\delta=11.27$ (s, 1H, NH), 10.99 (s, 2H, NH), 8.28 (s, 1H, NH), $7.24-7.44$ (m, 5H, arom. H), 4.88 (s, 1H, 5-H). - MS: $m / z=251[\mathrm{M}+\mathrm{H}]^{+}$.

\section{5-(2-Hydroxyphenyl)-1,3,5,6,8-pentahydro-7-thioxo- pyrimido[4,5-d]pyrimidine-2,4-dione $(\boldsymbol{6} \boldsymbol{b})$}

White solid; m.p. 200-202 ${ }^{\circ} \mathrm{C}$; anal. calcd. for $\mathrm{C}_{12} \mathrm{H}_{10} \mathrm{~N}_{4} \mathrm{O}_{3} \mathrm{~S}$ : C 49.65, H 3.44, N 19.31; found: C 49.59, H 3.36, N 19.47. - IR (nujol): $v=3446,3222,3080$,
1697, 1650, $1582 \mathrm{~cm}^{-1} .-{ }^{1} \mathrm{H}$ NMR $\left(300 \mathrm{MHz}, \mathrm{CDCl}_{3}+\right.$ [D ${ }_{6}$ ]DMSO): $\delta=11.26$ (s, 1H, NH), 10.97 (s, 2H, NH), 8.29 (s, 1H, NH) $7.23-7.30$ (m, 4H, arom. H), 4.89 (s, 1H, 5-H). MS: $m / z=291[\mathrm{M}+\mathrm{H}]^{+}$.

\section{5-(4-Methoxyphenyl)-1,3,5,6,8-pentahydro-7-thioxo-} pyrimido[4,5-d]pyrimidine-2,4-dione (6c)

Yellow solid; m.p. $>300{ }^{\circ} \mathrm{C}$; anal. calcd. for $\mathrm{C}_{13} \mathrm{H}_{12} \mathrm{~N}_{4} \mathrm{O}_{3} \mathrm{~S}$ : C 51.31, H 3.94, N 18.42; found: C 51.37, $\mathrm{H}$ 3.80, N 18.35. - IR (nujol): $v=3452,3211,1720$, 1698, 1655, $1560 \mathrm{~cm}^{-1}$. - ${ }^{1} \mathrm{H}$ NMR $\left(300 \mathrm{MHz}, \mathrm{CDCl}_{3}+\right.$ [D 6 DMSO): $\delta=11.29$ (s, 1H, NH), 10.99 (s, 2H, NH), $8.31(\mathrm{~s}, 1 \mathrm{H}, \mathrm{NH}), 7.22(\mathrm{~d}, J=7.0 \mathrm{~Hz}, 2 \mathrm{H}$, arom. H), 7.16 $(\mathrm{d}, J=7.0 \mathrm{~Hz}, 2 \mathrm{H}$, arom. H), $4.88(\mathrm{~s}, 1 \mathrm{H}, 5-\mathrm{H}), 3.83$ (s, 3H, $\left.\mathrm{OCH}_{3}\right) .-\mathrm{MS}: m / z=305[\mathrm{M}+\mathrm{H}]^{+}$.

5-(p-Chlorophenyl)-1,3,5,6,8-pentahydro-7-thioxopyrimido[4,5-d]pyrimidine-2,4-dione (6d)

White solid; m.p. $278{ }^{\circ} \mathrm{C}$ (d); anal. calcd. for $\mathrm{C}_{12} \mathrm{H}_{9} \mathrm{~N}_{4} \mathrm{O}_{2} \mathrm{SCl}$ : C 42.17, H 3.16, N 19.68; found: C 42.15, H 3.26, N 19.57. - IR (nujol): $v=3457,3201,1713$, 1700, 1658, $1560 \mathrm{~cm}^{-1} .-{ }^{1} \mathrm{H}$ NMR $\left(300 \mathrm{MHz}, \mathrm{CDCl}_{3}+\right.$ [D $\mathrm{D}_{6}$ ]DMSO): $\delta=11.29$ (s, 1H, NH), 10.98 (s, 2H, NH), 8.26 (s, $1 \mathrm{H}, \mathrm{NH}), 7.59(\mathrm{~d}, J=8.0 \mathrm{~Hz}, 2 \mathrm{H}$, arom. H), 7.21 (d, $J=$ $8.0 \mathrm{~Hz}, 2 \mathrm{H}$, arom. H), 4.83 (s, 1H, 5-H). - MS: $m / z=291$ $[\mathrm{M}+\mathrm{H}]^{+}$.

\section{5-(1,3-Benzodioxol-5-yl)-1,3,5,6,8-pentahydro-7-thioxo- pyrimido[4,5-d]pyrimidine-2,4-dione (6e)}

Yellow solid; m.p. $>260{ }^{\circ} \mathrm{C}$ (d); anal. calcd. for $\mathrm{C}_{13} \mathrm{H}_{10} \mathrm{~N}_{4} \mathrm{O}_{4} \mathrm{~S}$ : C 49.05, H 3.14, N 17.61; found: C 49.11, H 3.23, N 17.54. - IR (nujol): $v=3480,3273,3090,1760$, 1696, 1635, $1575 \mathrm{~cm}^{-1} .{ }^{1} \mathrm{H}$ NMR $\left(300 \mathrm{MHz}, \mathrm{CDCl}_{3}+\right.$ [D $\mathrm{D}_{6}$ ]DMSO): $\delta=11.31(\mathrm{~s}, 1 \mathrm{H}, \mathrm{NH}), 10.95(\mathrm{~s}, 2 \mathrm{H}, \mathrm{NH}), 8.26$ (s, 1H, NH), 7.11 (s, 1H, arom. H), 6.87 (d, $J=8.0 \mathrm{~Hz}, 1 \mathrm{H}$, arom. H), 6.69 (d, $J=8.0 \mathrm{~Hz}, 1 \mathrm{H}$, arom. H), $5.95(\mathrm{~s}, 2 \mathrm{H}$, $\left.\mathrm{OCH}_{2} \mathrm{O}\right), 4.90(\mathrm{~s}, 1 \mathrm{H}, 5-\mathrm{H}) .-\mathrm{MS}: m / z=319[\mathrm{M}+\mathrm{H}]^{+}$.

\section{5-(2-Thienyl)-1,3,5,6,8-pentahydro-7-thioxo-pyrimido} [4,5-d]pyrimidine-2,4-dione $(\mathbf{6 f})$

Yellow solid; m.p. $240-242{ }^{\circ} \mathrm{C}$; anal. calcd. for $\mathrm{C}_{10} \mathrm{H}_{8} \mathrm{~N}_{4} \mathrm{O}_{2} \mathrm{~S}_{2}$ : C 42.85, H 2.85, N 20.00; found: C 42.76, H 2.88, N 20.08. - IR (nujol): $v=3472,3146,3062,1756$, 1683, 1655, $1558 \mathrm{~cm}^{-1} .{ }^{1} \mathrm{H}$ NMR $\left(300 \mathrm{MHz}, \mathrm{CDCl}_{3}+\right.$ [D 6 DMSO): $\delta=11.28$ (s, 1H, NH), 10.91 (s, 2H, NH), 8.42 $(\mathrm{d}, J=4.6 \mathrm{~Hz}, 1 \mathrm{H}$, thienyl-H), 8.31 (s, $1 \mathrm{H}, \mathrm{NH}), 8.18$ (d, $J=$ $3.0 \mathrm{~Hz}, 1 \mathrm{H}$, thienyl-H), $7.48(\mathrm{t}, 1 \mathrm{H}$, thienyl-H), 4.89 (s, $1 \mathrm{H}$, 5-H). - MS: $m / z=304[\mathrm{M}+\mathrm{H}]^{+}$.

\section{Acknowledgement}

The authors acknowledge the financial assistance by the Council of Scientific and Industrial Research (CSIR), New Delhi. 
[1] a) M. Kidwai, S. Saxena, M. K. R. Khan, S. S. Thukral, Bioorg. Med. Chem. Lett. 2005, 15, 4295-4298; b) P. A. Grieco, Organic Synthesis in Water, Chapman \& Hall, New York, 1998, 250, p. 1-41; c) P. A. Grieco, E. B. Brands, S. Mc. Cann, J. D. Clark, J. Org. Chem. 1989, 54, 5489-5491.

[2] D. C. Rideout, R. Breslow, J. Am. Chem. Soc. 1980, $102,7816-7817$.

[3] a) K. A. Hansford, V. Zanzarova, A. Dorr, W. D. Lubell, J. Comb. Chem. 2004, 6, 893 -898; b) A. R. Katritzky, Chem. Rev. 2004, 104, 2125-2182.

[4] a) G. Heckmann, T. Bach, Angew. Chem. Int. Ed. 2005, 44, 1199-1201; b) E. C. Taylor, R. J. Knopf, R.F. Meyer, A. Holmes, M. L. Hoefle, J. Am. Chem. Soc. 1960, 82, $5711-5718$.

[5] A. M. Doherty, Ann. Rep. Med. Chem. 2004, 39, $335-$ 368.

[6] Review: C. O. Kappe, Acc. Chem. Res. 2000, 33, 879888.

[7] M. Kidwai, S. Saxena, M. K. R. Khan, S. S. Thukral, Europ. J. Med. Chem. 2005, 40, 816-819.

[8] a) J.D. Brown in Comprehensive Heterocyclic Chemistry, Vol. 3 (Eds.: A. R. Katritzky, C. W. Rees), Pergamon, Oxford, 1984, p. 57; b) E. D. Clercq, R. Beraaerts, J. Biol. Chem. 1987, 262, 14905 - 14911.

[9] A. A. Ramsey, US 3830812, 1974; Chem. Abstr. 1974, 81,136174
[10] N. Kitamura, A. Onishi, EU 163599, 1984; Chem. Abstr. 1984, 104, 186439.

[11] R. B. Tenser, A. Gaydos, K. A. Hay, Antimicrob. Agents Chemother. 2001, 45, 3657-3659.

[12] a) P. Sharma, N. Rane, V. K. Gurram, Bioorg. Med. Chem. Lett. 2004, 14, 4185-4190; b) J. Cieplik, J. Pluta, O. Gubrynowicz, Bull. Chim. Farm. 2003, 142, $146-150$.

[13] J. P. De la Cruz, T. Carrasco, G. Ortega, De La Cuesta F. Sanchez, Lipids 1992, 27, $192-194$.

[14] V. J. Ram, A. Goel, S. Sarkhel, P. R. Maulik, Bioorg. Med. Chem. 2002, 10, 1275-1280.

[15] K. Hirota, Y. Kitade, H. Sajiki, Y. Maki, J. Chem. Soc., Perkin Trans. 1 1990, 123-125.

[16] S. K. Srivastava, W. Haq, P. M. S. Chauhan, Bioorg. Med. Chem. Lett. 1999, 9, $965-966$.

[17] H. Wamhoff, J. Muhr, Synthesis 1998, 919-926.

[18] D. Prajapati, A. J. Thakur, Tetrahedron Lett. 2005, 46, $1433-1436$.

[19] K. Hirota, Y. Kitade, H. Sajiki, Y. Maki, Synthesis 1984, $589-590$.

[20] a) M. Kidwai, K. Singhal, Synth. Comm. 2006, 36, 1887-1891; b) M. Kidwai, K. Singhal, R. Thakur, Lett. Org. Chem. 2005, 2, 419-424; c) M. Kidwai, S. Rastogi, R. Thakur, S. Saxena, Z. Naturforsch. 2004, $59 b, 606-608$. 